\title{
LA PESCA FLUVIAL COMO RECURSO ECONÓMICO EN ÉPOCA IBÉRICA TARDÍA: UN EJEMPLO PROCEDENTE DE LOS CASTELLONES DE CÉAL (HINOJARES, JAÉN) (1)
}

\author{
RIVER FISHING AS AN ECONOMIC RESOURCE IN LATE IBERIAN TIMES: \\ AN EXAMPLE FROM THE SITE OF LOS CASTELLONES DE CÉAL (HINOJARES, JAÉN)
}

VICTORINO MAYORAL HERRERA (*)

TERESA CHAPA BRUNET $(*)$

JUAN PEREIRA SIESO $(* *)$

ANTONIO MADRIGAL BELINCHÓN $(*)$

\section{RESUMEN}

En este trabajo se describen una serie de láminas enrolladas de plomo aparecidas en el nivel iberorromano de Castellones de Céal y que habitualmente son empleadas como lastres en redes de pesca. Se deduce de ello el empleo de la pesca fluvial como recurso económico, revisándose los ejemplos conocidos de esta actividad en el registro arqueológico.

\begin{abstract}
This paper describes the finding of a group of folded lead sheets in the Ibero-roman level at Castellones de Céal. As they have been traditionally used as weights for fishing nets, the practice at the site of river fishing as an economic resource is inferred. Some other archaeological examples of this activity are also examined.

Palabras clave: Pesca fluvial. Redes de pesca. Plomos de red. Cultura ibérica. Fase romana republicana. Economía ibérica.

(*) Dpto. de Prehistoria. Universidad Complutense. 28040 Madrid. Correo electrónico: tchapa@eucmax.sim.ucm.es.

(**) Área de Prehistoria. Universidad de Castilla-La Mancha. Campus de Toledo. Plaza de Padilla 4. 41071 Toledo.

El artículo fue remitido en su versión final el 28-I-2000.

(1) Este trabajo se encuadra en el Proyecto "Estudio del poblamiento ibérico en el valle del Guadiana Menor desde la perspectiva de la Arqueología del Paisaje" (DGES PB98/0775).
\end{abstract}

Keywords: River fishing. Fishing nets. Net lead weights. Iberian Culture. Roman Republican phase. Iberian Economy.

\section{INTRODUCCIÓN}

El conocimiento de la vida económica concreta de los poblados ibéricos es aún muy escaso, dado que ni los objetivos de la investigación ni a menudo las condiciones de conservación han permitido obtener una información suficientemente completa. De todas las labores que se llevaban a cabo en los pequeños asentamientos antiguos hay algunas, además, que resultan aún más difíciles de detectar, dado que implican el uso de un instrumental no duradero y la obtención de unos recursos cuya preservación puede considerarse como un hecho excepcional. Este es el caso de la pesca, que debió practicarse habitualmente en los ríos, y de la que sin embargo rara vez encontramos evidencias arqueológicas. El acceso a este recurso ha sido constante en la Historia, y supone tanto actividades especializadas como complementarias a las economías de carácter campesino del tipo que podemos esperar en un poblado ibérico de pequeñas dimensiones como el de Castellones de Céal.Asimismo, la práctica de la pesca implica todo un conjunto de enseres y de actividades que se engranan en las prácticas sociales, y de las que apenas sabemos nada hasta el momento. 


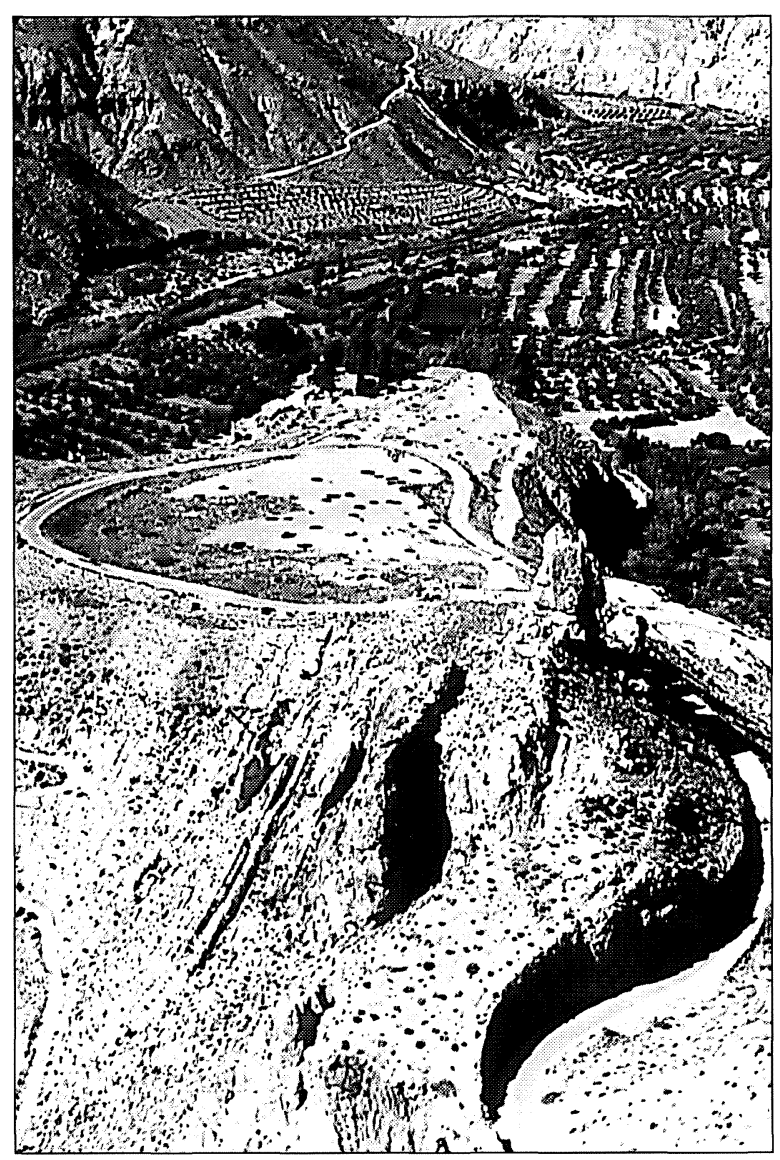

Lám. I. Los Castellones de Céal. Vista general del poblado en la confluencia de los ríos Céal y Guadiana Menor.

El asentamiento de Los Castellones (Lám. I) es bien conocido en la bibliografía por los trabajos desarrollados en los años cincuenta (Fernández Chicarro, 1955a) y por nuevas campañas (Chapa et alii, 1998). Consiste en un pequeño poblado, de menos de $2 \mathrm{Ha}$, situado en el estrecho y difícil pasillo que une el Alto Guadalquivir con las altiplanicies granadinas. Ocupa un cerro protegido de forma natural por bruscos acantilados formados por la erosión de dos cursos fluviales que se unen al pie del yacimiento: el Guadiana Menor y el arroyo de Céal. El hábitat (Fig. 1) se ciñe a la parte superior del promontorio, emplazándose la necrópolis a una cota más baja en la ladera norte. Tras una breve ocupación que se remonta probablemente al siglo VII-VI a.C., el poblado se refunda a finales del siglo V a.C., y permanecerá activo hasta algo antes del cambio de Era, mostrando por tanto los cambios correspondientes a la progresiva ocupación romana.

\section{CARACTERÍSTICAS DEL HALLAZGO}

La existencia de una red de pesca se deduce de la presencia de un conjunto formado por 44 láminas enrolladas de plomo más los restos parcialmente fundidos de otras 20. Estos cilindros son habitualmente utilizados como lastre de redes tanto en ejemplos antiguos como modernos. Son piezas bastante uniformes, con longitudes entre 4.3 y $3.2 \mathrm{~cm}$, situándose la mayoría de ellos entre 3.5 y $3.7 \mathrm{~cm}$. Su peso oscila entre los 25 y los $30 \mathrm{~g}$, lo que implica un lastre total de aproximadamente $1.800 \mathrm{~g}$. Junto a ellos apareció una piedra con perforación $(10 \times 12 \mathrm{~cm})$ que pudo cumplir las funciones de borlón o lastre más grueso, así como una fusayola (Fig. 2). Todo ello pertenecía a un patio-almacén semicubierto, situado en el espacio A de la vivienda 1, en la parte más alta del poblado (Mayoral, 1996). La acumulación de materiales era aqui muy notable, destacando los recipientes cerámicos entre los que se cuentan dos ánforas vinarias de tipo greco-itálico y Dressel 1-A, y cuatro ánforas ibéricas. Los plomos se encontraron caidos entre las ánforas como resultado del desplome del chamizo que las cobijaba. La posición en hilera de algunos de ellos sugiere que la red estuviera montada y colgada de la pared en el momento del violento incendio que arrasó la estructura.

El uso del plomo resulta frecuente en este contexto, aprovechándose para tapaderas o recipientes, pero transformado en goterones informes en aquellos lugares en los que el fuego fué más intenso. Su fundición, fácil de conseguir, pudo efectuarse en el mismo lugar, si tenemos en cuenta la presencia de un recipiente de tipo mortero con pico vertedor, en el que se advirtieron residuos de plomo (Mayoral, 1996: 239). Junto a todo ello se recuperaron instrumentos de muy diverso carácter, tanto de uso agrícola como de caza, lo que confirma el carácter polifuncional de este espacio.

\section{SISTEMAS DE PESCA FLUVIAL TRADICIONAL}

El conocimiento de las técnicas pesqueras en la antigüedad ha recabado el interés de muchos investigadores, siendo habitual en las primeras monografías el referirse a los tiempos prehistóricos junto a la descripción de los sistemas de pesca entre los pueblos "primitivos". Junto a la obra de 


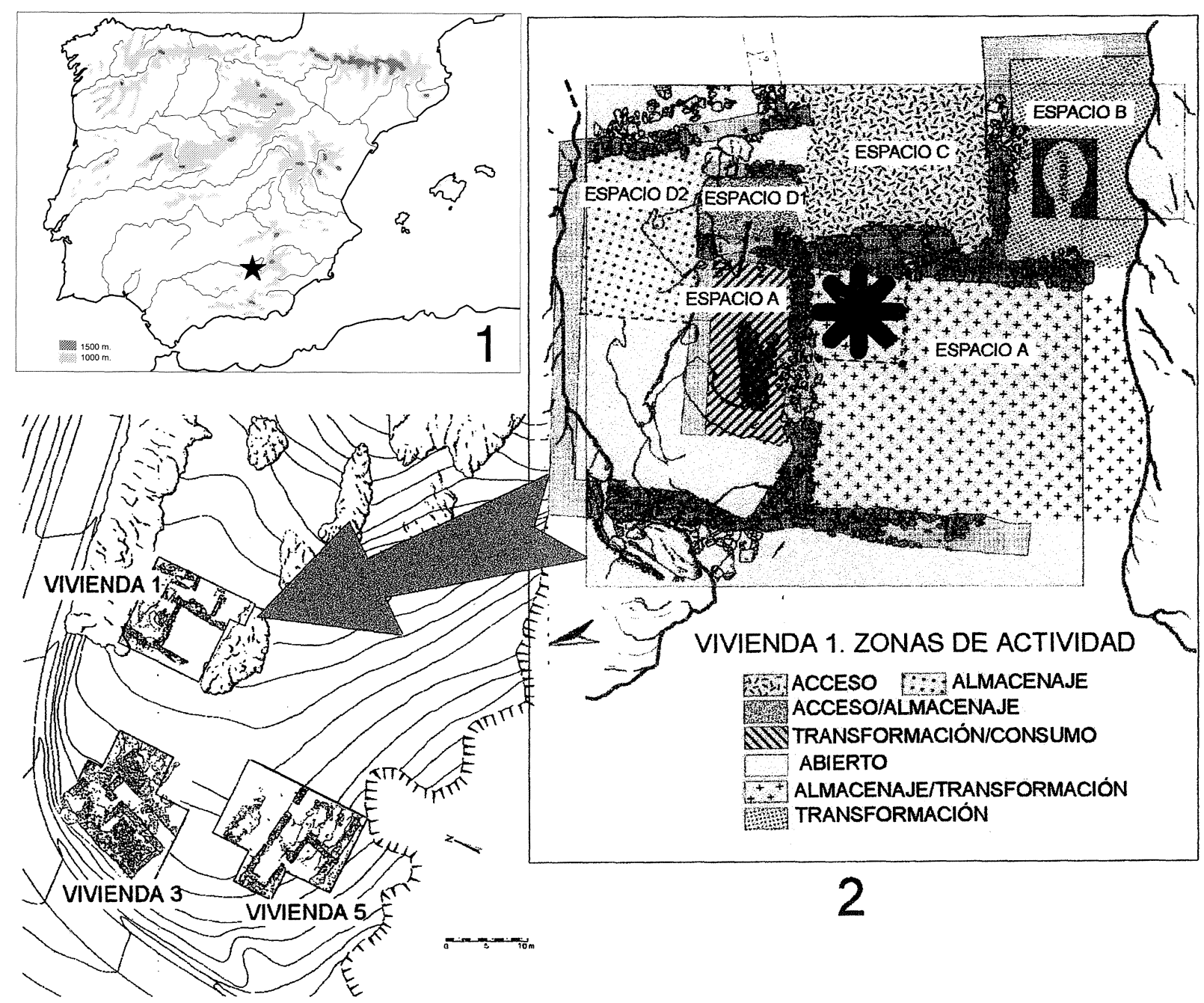

Fig. 1. Los Castellones de Céal: 1. Situación del asentamiento. 2. Plano de las habitaciones del poblado y detalle de la vivienda 1 .

Rau (1884) y la ya clásica de Radcliffe (1926), verdadero compendio sobre el mundo antiguo, pueden citarse las de Gruvel (1928), Thomazi (1947), Vega Ferreira (1968) o Gunda (1984), orientada esta última a la evidencia etnográfica. Estrictamente arqueológicos son los breves apuntes de Clark (1948) o los más recientes estudios sobre las primeras evidencias de la pesca en Europa (Cleyet-Merle, 1990; Le Galle, 1984; Brinkhuizen y Clason, 1986; Casteel, 1976).

Los autores griegos y romanos son una fuente primordial para comprender tanto la importancia de la pesca en esas épocas como los sistemas empleados en esta actividad, si bien las noticias que poseemos se ciñen al entorno marítimo, ya sea desde embarcaciones o a nivel de costa.
Sin embargo, el conocimiento de las artes de pesca tradicionales, tanto en Francia como en España, se apoya especialmente en obras de la época ilustrada como las de Duhamel (1769-1779) o Sáñez Reguart (1791). En ellas se describen cuidadosamente los sistemas entonces en uso, tanto en el campo marítimo como el fluvial, con ilustraciones precisas y de gran calidad (Figs. 3 y 4.1). Estas monografías son muy utilizadas actualmente para interpretar el material arqueológico de diversos yacimientos (Sternberg, 1995, con referencias sobre el área francesa).

La bibliografía específica sobre la pesca fluvial en época reciente no es demasiado abundante en la Península Ibérica. Resulta fundamental en este sentido el libro de Pardo (1950), que analiza la evolu- 


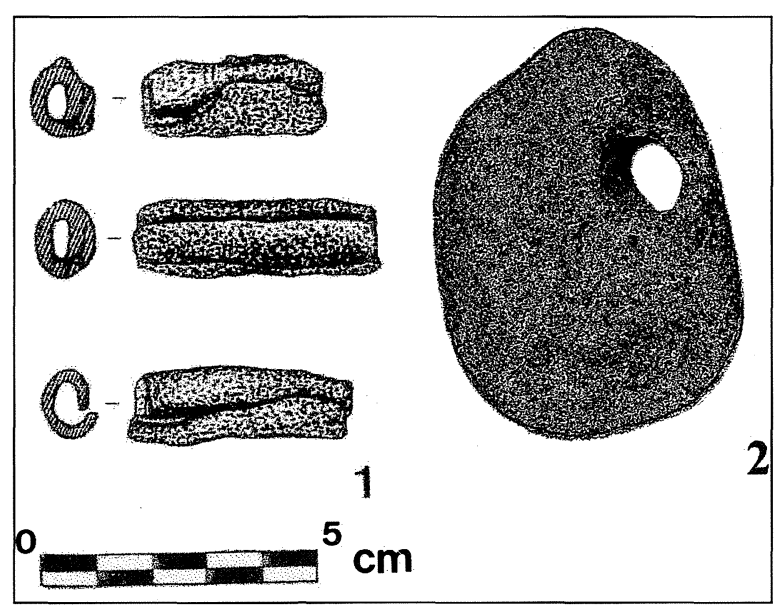

Fig. 2. Los Castellones de Céal: 1. Una muestra de las láminas enrolladas de plomo. 2. Lastre de piedra.

ción histórica incluyendo algunas notas sobre épocas prerromanas. Es también interesante la obra de Merino (1986), aunque más localizada en el País Vasco y con un lógico interés prioritario en la pesca marítima. De carácter más técnico y descriptivo son los trabajos de Bas (1955), Melcón (1964), Navas (1945) o Puche (1959). En el campo de la Etnografía deben resaltarse los trabajos de Lorenzo Fernández (1979), Ladra Fernández (1998), Ladra Fernández y Pereiras Magariños (e.p.), Agudo Torrico (1992) y Mora Aliseda (1988), que aportan interesante información sobre el uso de la pesca fluvial como recurso económico en las áreas gallega, andaluza y extremeña respectivamente, y que estudian con detenimiento la elaboración de redes que probablemente tienen mucho en comun con el ejemplo que presentamos.

La pesca de anzuelo ha sido seguramente el sistema más habitual para la obtención de peces en los cursos fluviales, documentándose numerosos anzuelos de bronce en diversos yacimientos iberorromanos peninsulares (Gracia Alonso, 19811982). Sin embargo, como hemos indicado, en Castellones de Céal aparecen plomos que deben interpretarse como pesas para una red. Hay dos tipos principales de redes empleadas tradicionalmente en la pesca fluvial y en las que se usan este tipo de lastres:

a) El Esparavel o Tarraya (Fig. 3). Es un sistema muy empleado, tanto en su variedad comun como en la de anillo, ya que puede ser manipulado por una sola persona. El pescador se situa en la orilla, en una embarcación o dentro del agua, pero siempre en cursos poco profundos, ya que es preci- so detectar la presencia del pez antes de lanzar el arte. Su elaboración requiere un proceso complicado, puesto que son necesarias doce docenas de mallas enlazadas en círculo formando una corona, rematada por una capa de doble tejido, y la llamada bolsa, que forma el vértice central y que es el punto en el que queda atrapado el pescado cuando se recoge el esparavel. El tamaño, tanto de la red como de la malla, depende del medio en el que se pesca, del tamaño de los peces que se espera obtener y de la propia fuerza y capacidad de manipulación del pescador. La pieza se remata en la base con cuerda o hilos por los que se hacen pasar los plomos cilíndricos. Estos deben ser más numerosos y algo más largos si el esparavel es grande, quedando entre uno y otro una distancia no mayor de $10 \mathrm{~cm}$. La red debe arrojarse con destreza y asegurarse a la muñeca del pescador mediante un cordel que servirá igualmente para recuperarla con su contenido de peces. Además de un uso individual mediante lanzamiento puede practicarse también la pesca por rastreo, sujetando sus bordes dos personas -una en cada orilla del río- y estirando otra la red desde atrás. Este sistema suele emplear también a otros pescadores que remueven con palos las orillas, dirigiendo los peces hacia la red a la vez que evitan que ésta se enganche en plantas y piedras. Esta pesca por rastreo fue descrita por Duhamel y recogida por Sáñez Reguart (1791) quien sin embargo no documenta su uso en España.

b) El Trasmallo (Fig. 4), que en su versión simplificada suele llamarse traviesa. Se trata de una red rectangular formada por tres paños de distinta luz de malla. Su elaboración requiere tejer primero el manto o lienzo de la red, reforzándolo después mediante las albitranas o tejido de ajuste. La pieza se remata en la parte superior e inferior con los llamados cordeles, trallas o relingas. En la de arriba se enfilan los corchos que permitirán mantener la línea de flotación, mientras que en la de abajo se situarán los plomos cilíndricos, cuyo número variará segun el tamaño total de la red. Para arrojarla y mantenerla fija se le aplica un lastre de mayor tamaño que suele ser una piedra, perforada o no, o algun otro elemento de peso. Si la red es grande, se le añaden además lastres adicionales denominados borlones, rítmicamente dispuestos en el cordel inferior. Pueden ser bloques de barro cocido horadados, de forma muy parecida a las pesas de telar, pero también pueden emplearse simples piedras atadas. La longitud de las redes depende de la anchura del río o 


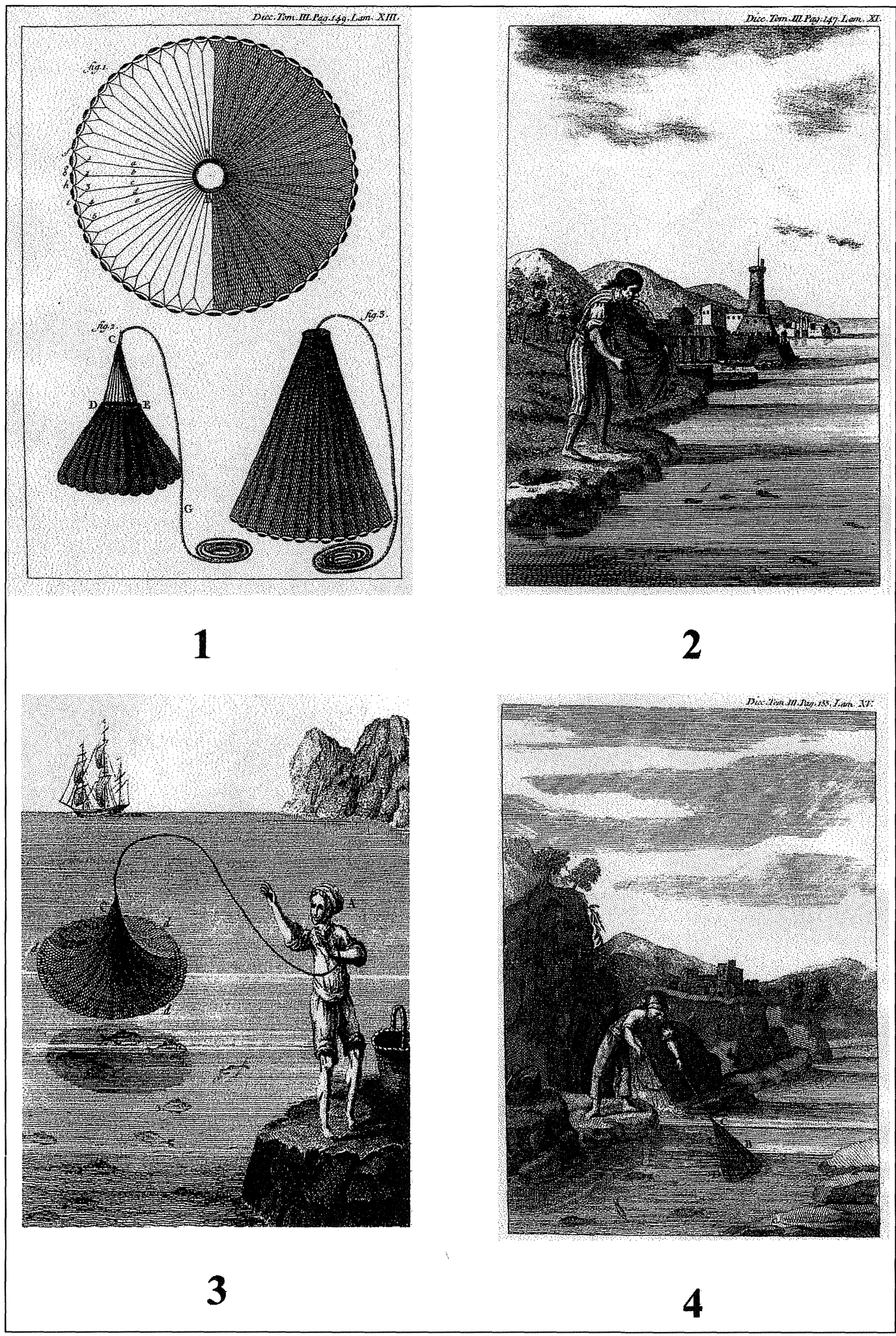

Fig. 3. 1. Estructura de un esparavel o tarraya. 2 a 4. Preparación, lanzado y recogida de la red (según Sáñez Reguart, 1791). 


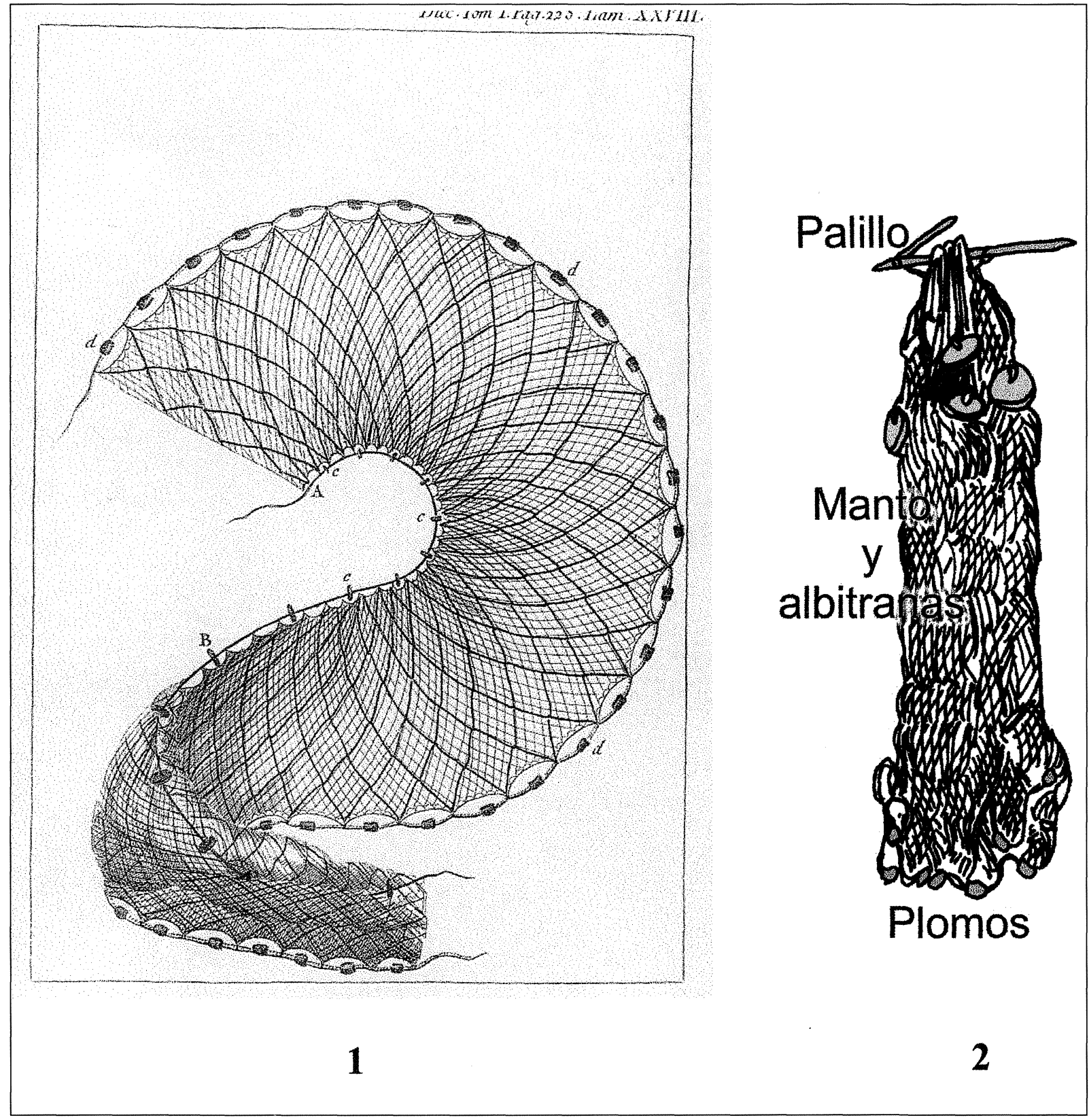

Fig. 4. 1. Estructura del trasmallo (según Sáñez Reguart, 1791). 2. Trasmallo recogido (según Mora Aliseda, 1988).

de la zona que se quiera abarcar. En época reciente se documentan redes de $24 \mathrm{~m}$ en el Guadiana, y de $18 \mathrm{~m}$ en el Tajo (Mora Aliseda, 1988: 9), siendo su altura aproximada de $1.50 \mathrm{~m}$. Este sistema permite una pesca activa -removiendo las orillas y obligando a los peces a dirigirse hacia la red-o pasiva, montando el arte y dejando pasar un tiempo hasta recoger los pescados que hayan caído en ella, incluyendo si acaso algo de cebo.

\section{EJEMPLOS ARQUEOLÓGICOS DE PESCA CON RED}

El uso de redes con lastres de plomo se remonta al menos al segundo milenio a.C., sustituyendo a los pesos de piedra que eran habitualmente empleados con este fin. Las ventajas aportadas por las piezas de plomo se refieren a su mayor regularidad y peso por unidad. Al tratarse de simples planchas 
hechas en un molde que se pliegan sobre el propio cordel, se pueden conseguir muchas del mismo tamaño y peso, lo que asegura un comportamiento uniforme de la parte inferior de la red. Por otra parte, como señala Powell (1996: 106), un plomo supone cinco veces el peso de una piedra de igual tamaño, por lo que resultan necesarias menos piezas, y éstas se adaptan mejor a la cuerda.

Esta autora recoge todos los hallazgos conocidos de pesas de plomo en la Grecia micénica, destacando los ejemplos de redes procedentes de los pecios de Cabo Gelindoya y, sobre todo, Ulu Burun. Aquí fueron recuperados 77 plomos en tres conjuntos, uno de los cuales apareció dentro de un jarro, por lo que Pulak (1988: 107) consideró que se trataba de una red que figuraba como mercancía en el barco. Sin embargo, su asociación a una lámpara de aceite hace pensar a Powell (1996: 120) que se trata de una red empleada en la pesca nocturna. Entre los hallazgos de este pecio apareció un gran peso piramidal de plomo que ha sido interpretado como lastre de red o como un ancla (Pulak, 1988: 32-33). Los hallazgos de este tipo no se limitan a contextos marítimos, sino que los conjuntos de plomo pueden ser incluidos en los ajuares funerarios, casi todos ellos fechados en la última fase de la Edad del Bronce en el Egeo (LBA). Su abundancia relativa debe entenderse en un contexto en el que la explotación de las minas de plata está en su apogeo, lo que provoca la producción de grandes cantidades de plomo que se comercializa y emplea de diversas maneras (Stos-Gale y MacDonald, 1991: 268-9).

De la misma época que las piezas de Céal y en un área más próxima hay que citar los interesantísmos hallazgos de Lattes (Lattara), un establecimiento costero del sureste francés en el que se practicó activamente tanto la pesca marítima como lagunar (Fig. 5.1). El estudio en extensión del yacimiento ha permitido recuperar una información global sobre las actividades pesqueras, ya que se conservan tanto elementos correspondientes a las artes empleadas -plomos de red, anzuelos- como de los instrumentos necesarios para elaborarlas -agujas, lanzaderas-, así como abundantes restos de ictiofauna y textos antiguos que hacen referencia a este lugar. Todo ello permite ofrecer una visión de conjunto sobre las estrategias de pesca y sus transformaciones a lo largo de la vida de este lugar entre los siglos III y I a.C. (Feugère, 1992; Sternberg, 1995). El conjunto principal de plomos proviene de la manzana 4-sur, y se fecha en los inicios

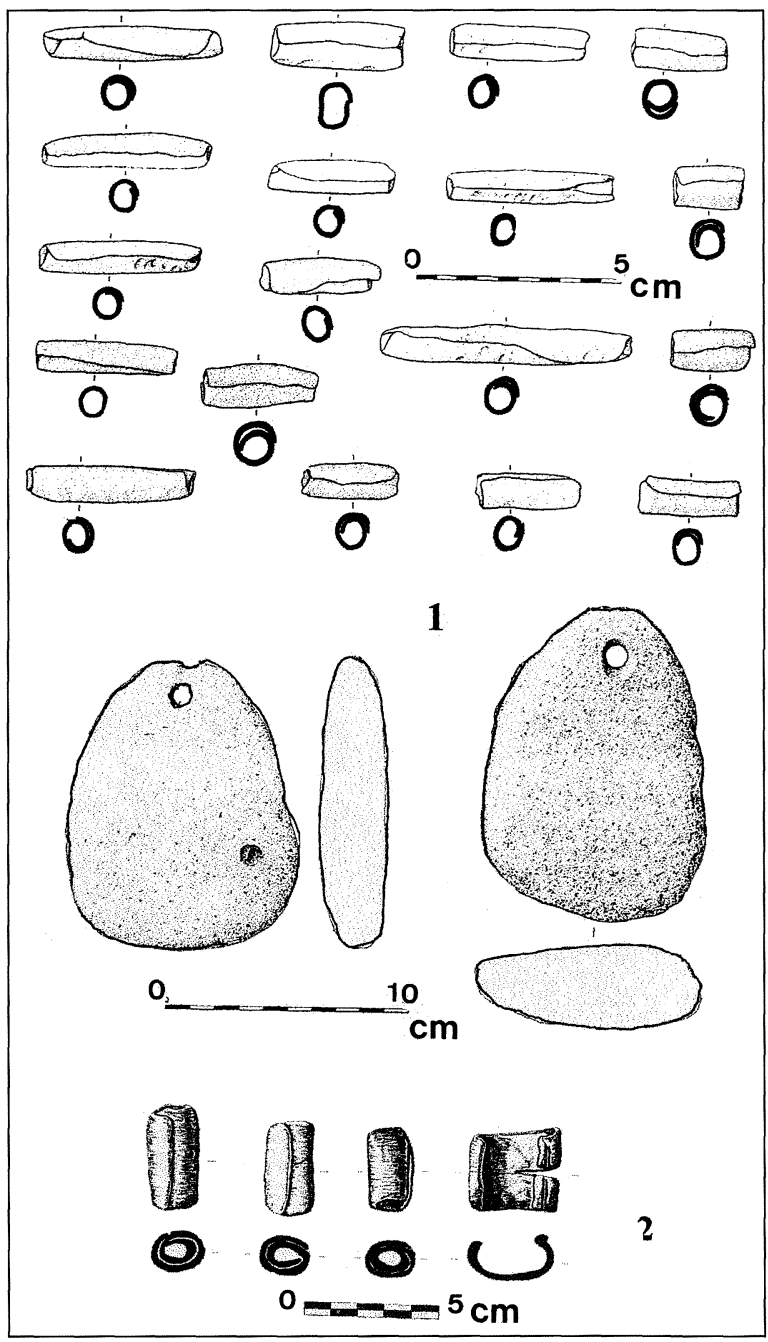

Fig. 5. 1. Lastres de plomo y de piedra de Lattes (según Feugère, 1992). 2. Plomos de Cancho Roano (según Celestino, 1996).

del siglo II a.C. (Py, 1991: 380, fig. 9). Es muy similar a la evidencia de Céal, y va ligada también a la presencia de pesas de piedra con perforación, pero su número es inferior, por lo que los autores opinan que hay que entenderlo como un depósito de repuesto o como la prueba de que la muestra arqueológica implica una pérdida considerable respecto a su categoría inicial. Otros conjuntos cerrados, como el del pecio de Porto Vecchio, aportan un número y un peso total de los plomos muy aproximado al de Céal, aunque algo menor (Feugère, 1992: 152).

En la Península Ibérica son aún pocos los testimonios arqueológicos relacionados con la pesca, y en general ceñidos al entorno costero. Es excepcio- 
nal la conservación en este medio de largas estructuras formadas por estacas de madera que conducían a los peces hacia una red de tipo nasa en un entorno de laguna próximo a la línea de costa, como la documentada en Silvade (Portugal) (Alves et alii, 1988-89). Por su parte, los plomos cilíndricos que pueden ser considerados como pesos de red apenas han sido recopilados o estudiados. Sin embargo, estas piezas son frecuentes en las colecciones debido a su fácil localización visual o mediante detectores de metales, sin que haya por tanto contextos arqueológicos fiables. Un caso especial es el del palacio-santuario de Cancho Roano, en Zalamea de la Serena, donde aparecieron piezas de estas características que los excavadores interpretan de manera diversa. Un grupo de piezas de pequeño tamaño encontradas en distintas habitaciones fueron consideradas como lastres para vestimentas o piezas de tela (S. Celestino, com.pers.). Otro grupo (Fig. 5.2), de dimensiones algo mayores, apareció en una de las habitaciones perimetrales del sector oeste, y es posible que se emplearan como pesos de red (Celestino, 1996: 86). En todo caso, queda claro que el empleo de este tipo de piezas es aquí muy anterior al que se constata en Céal o en Lattes, ya que en el yacimiento extremeño pueden remontarse al siglo $\mathrm{V}$ a.C.

En época ibérica tardía aún no se ha sistematizado el instrumental dedicado a la pesca, probablemente porque antes de la presencia de lastres de plomo se emplearían otros materiales, como piedras, "fusayolas" o pondera, lo que las hace prácticamente indetectables en el primer caso, e indistinguibles de los elementos dedicados a labores de tejido en el segundo y tercero. La iconografía nos puede servir de ayuda a través de ejemplos que ya rastreó Pardo (1950: fig. 12), y de los que destacamos la gran vasija del Departamento 15 del Tossal de San Miguel de Líria, en la que se representa una embarcación con posibles aparejos y un personaje sujetando una red con una mano y un hilo con un pez enganchado en su extremo (Ballester et alii, 1954: 47; Bonet, 1995, fig. 44). Es difícil saber hasta qué punto la ideología de la Alta Andalucía compartía las valoraciones religiosas que los peces parecen tener en otros lugares del Mediterráneo y en especial en la propia zona valenciana (Aranegui, 1996).

Las mismas artes se han empleado en fases más recientes. Un caso documentado arqueológicamente es el de la Cueva del Peñascal de los Infiernos, en Liétor (Albacete), donde apareció un conjunto de

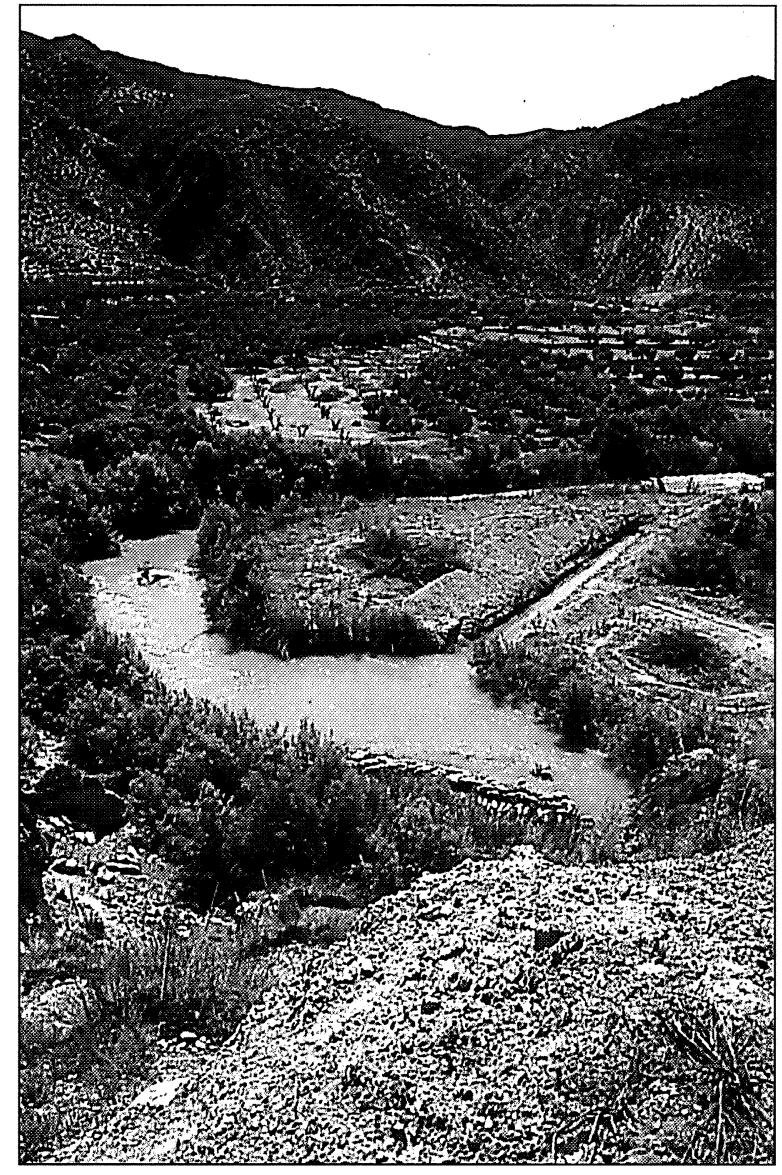

Lám. II. Vado sobre el río Guadiana Menor.

60 pequeños cilindros de plomo y 3 flotadores de madera que sus excavadores (Navarro Palazón y Robles Fernández, 1996: 63) interpretaron sin lugar a dudas como evidencia de un trasmallo o red lineal. El conjunto fue fechado entre los siglos X y XI d.C.

Los ejemplares de trasmallos y esparaveles conservados en el Museo Nacional deAntropología de Madrid confirman que estos sistemas tradicionales permanecen casi invariables hasta épocas muy recientes, utilizando los mismos tipos de lastres que encontramos en los yacimientos arqueológicos. $\mathrm{La}$ muestra, procedente en su mayor parte del área valenciana, corresponde al instrumental empleado para la pesca en áreas de albufera, y estaba generalmente ligada al uso de embarcaciones. El tamaño de los cilindros de plomo varía en función de la entidad de la red, y su número en función de su longitud, pero en general siguen las mismas pautas que han sido descritas. 


\section{EL APROVECHAMIENTO PISCÍCOLA EN EL ENTORNO DE CASTELLONES DE CÉAL}

La excavación del yacimiento ibérico no ha proporcionado por el momento restos de ictiofauna, pero la caracterización ambiental y fluvial del entorno permiten evaluar las posibilidades de un recurso como la pesca en esa zona. El río principal es el Guadiana Menor (Lám. II), principal afluente y en realidad cabecera del Guadalquivir (Romero Díaz, 1989: 11). Su cuenca incluye como tributarios a los ríos Orce, Cúllar, Baza, Baúl, Fardes y Alicún por su margen izquierda, y a los ríos Guardal, Castril, Guadalentín, Turrilla, Céal y Toya por la derecha. El régimen hidrológico de estos ríos es pluvial, con un importante caudal en invierno y primavera y fuerte estiaje en los periodos secos. No obstante, la proximidad de las sierras de Cazorla, el Pozo o la Sagra, con su elevada altitud y el predominio de materiales calizos hace que en la margen derecha el estiaje sea mucho menos pronunciado, y que por lo tanto a la altura de Céal los cursos de agua permanezcan constantes (Picazo y Alba Tercedor, 1996: 156-157).

En la actualidad se documentan en esta zona varias especies endémicas susceptibles de pesca. Destacan Barbus sclateri (barbo gitano), que puede alcanzar los $40 \mathrm{~cm}$ y que evita las aguas estancadas o las excesivamente frías y rápidas; Chondrostoma polylepis (boga), de unos $30 \mathrm{~cm}$, que ocupa los tramos medios de los ríos y busca aguas limpias en los cauces altos para la puesta; Leuciscus pyrenaicus (cacho o cachuelo), menor de $30 \mathrm{~cm}$ y que se adapta a medios fluviales variados; Tropidophoxinellus alburnoides (Calandino), especie pequeña, menor de $13 \mathrm{~cm}$, que se desarrolla en aguas de montaña (Doadrio et alii, 1991; Gómez Caruana y Díaz Luna, 1991).

En el siglo XIX, el Diccionario de Madoz (1988: 216), añade para el término de Pozo Alcón la presencia de anguilas en aguas calmas, siendo ésta una especie para la que es frecuente la pesca con red. También indica la abundancia de "exquisitas y abundantes" truchas en el Guadalentín, así como en el Guadiana Menor a la altura de Hinojares y Huesa, lo que incluye el entorno de Céal. Ambas especies han estado presentes en la zona hasta fechas muy recientes, en las que ha existido una sobrepesca, así como un cambio en las características de los ríos, con la construcción de presas y embalses, y con la incorporación de vertidos contaminantes de diverso tipo.
Aunque en estos momentos resulte difícil asegurar cuáles fueron las especies aprovechadas en época ibérica, podemos contar con datos de etapas muy anteriores, como la argárica, a través de los análisis de fauna de yacimientos como el CastellónAlto en Galera, cuyo río es uno de los que forman el curso medio-alto del Guadiana Menor. Aquí se han identificado (Milz, 1986: 90-91) restos correspondientes a barbo, que se ha mantenido hasta el presente, pero también de Tinca tinca (tenca) -dato que, de ser confirmado, resolvería la discusión sobre si es una especie local o introducida- y de Leuciscus cephalus (bagre), hoy limitado a la zona noreste peninsular.

Si recurrimos a las informaciones proporcionadas por los habitantes de la zona, que han desarrollado hasta hace relativamente poco tiempo una economía de carácter prácticamente autosuficiente, podemos advertir que el recurso a la pesca era ocasional entre la mayoría de los campesinos, pero que también existían pescadores especializados, y zonas con más dedicación que otras a la actividad pesquera. Para la pesca ocasional se han utilizado fundamentalmente dos sistemas: la pesca a mano y la pesca con caña. La primera podía realizarse de día o de noche. Durante el día, en zonas calmas de los ríos, se iban levantando aquellas piedras bajo las que podía cobijarse algún pez, hasta conseguir atrapar alguno que no tuviera una reacción suficientemente rápida. Por la noche una persona iluminaba desde la orilla un punto del río consiguiendo así un deslumbramiento momentáneo de los peces, que eran atrapados por el pescador. Para la pesca con caña, se aprovechaban los cañaverales que abundan en esta zona, escogiendo ejemplares de tamaño y flexibilidad adecuada y añadiéndoles el hilo, el peso y el anzuelo. Se han podido emplear también otros métodos para la pesca a mano que no requieren tanta habilidad, como echar en el agua cargas de dinamita o productos más naturales como cal o ciertas hierbas que, a decir de algunos, atontaban a los peces y permitían capturarlos fácilmente.

La pesca más ambiciosa, que a menudo tenía fines en parte comerciales, era realizada con redes. En el Guadiana Menor, a la altura del Fontanar, se utilizaban nasas que actuaban como trampas para el pescado en las que se disponía un cebo. Ocasionalmente se han empleado trasmallos, ajustando sus extremos a las orillas del río y dejándolo, por tanto, fijo. Los pescadores remontaban el curso fluvial hasta una cierta distancia, y con palos re- 
movían las aguas asustando a los peces para hacer que se dirigieran contra la red. En el cordel inferior del trasmallo se disponían plomos de las mismas características que los encontrados en el yacimiento, formados por láminas enrolladas de unos $6 \times 2 \mathrm{~cm}$, y espaciándolos aproximadamente 20 $\mathrm{cm}$. No existe, sin embargo, una tradición en la confección de estas redes, y las que se han utilizado han venido ya hechas en tejidos modernos como el nylon.

En los pueblos de la zona ha existido la posibilidad de comprar pescado fluvial fresco, que llegaba después del trabajo de los pescadores nocturnos, los cuales desarrollaban su actividad especialmente en el curso alto del Guadalquivir. No ha existido costumbre de hacer conserva de él, sino de consumirlo inmediatamente. Las elaboración de conservas se reservaba para los arenques, que venían de áreas mucho más lejanas.

\section{CONCLUSIÓN}

Como ya señaló Fletcher (1968: 50), la obtención de peces fué sin duda un recurso económico sistemáticamente explotado en zonas litorales durante la época ibérica, y no sólo en el entorno más relacionado con el mundo fenicio (Roselló y Morales, 1994; Frutos Reyes y Muñoz Vicente, 1996). Probablemente tuvo una importancia más limitada en el interior, donde debía vincularse a los cursos fluviales (2). Su carácter de actividad complementaria, el empleo de elementos perecederos para la elaboración de las artes de pesca, y la dificultad de conservación de la ictiofauna hace muy difícil su detección arqueológica. Incluso si preguntamos actualmente a los habitantes del lugar sobre sus actividades económicas, no se citará la pesca entre ellas (Chapa et alii, 1984; Fernández Rodríguez et alii, 1994). Sin embargo, ante cuestiones directas, hay muy pocos que no reconozcan haberla practicado de forma eventual o constante, empleando como hemos visto, sistemas diversos. La pesca fluvial resulta, por tanto, un recurso económico enmascarado pero que es preciso tener presente a la hora de valorar la subsistencia tanto en los asentamientos costeros como en los de interior.

(2) Un ejemplo esclarecedor lo proporciona el asentamiento de Numancia (Soria), donde además de la presencia de anzuelos y cerámicas con representación de peces (Jimeno Martínez et alii, 1999: 803) se ha podido confirmar el consumo de pescado mediante el análisis químico de los huesos humanos (Alfredo Jimeno, com. pers.).
La ocupación más tardía de Los Castellones de Céal revela una actividad acusada en la estrategia de comunicaciones entre las altiplanicies granadinas y el Alto Guadalquivir. Muchos de los materiales presentes en el "espacioA" del poblado son importados mostrando, como es natural en esta época, evidentes signos de romanización. Varios de ellos parecen estar en relación con el consumo del vino, no sólo por los envases anfóricos antes citados, sino por la presencia de un cazo de bronce, cubiletes y algún resto de cerámica megárica (Beltrán Lloris et alii, 1999). Además de estos objetos de procedencia lejana pueden señalarse también importaciones de otras áreas de la Península Ibérica, como un kálatos pintado de tipo Fontscaldes, una jarrita gris ampuritana y otras jarras idénticas a las procedentes del yacimiento almeriense de Villaricos.

La existencia del asentamiento debe ponerse en relación, por tanto, con su papel de apoyo en la red de comunicaciones, lo que le permite incorporar numerosos elementos foráneos. Sin embargo, también resulta evidente que su subsistencia se basó en una explotación intensa de su entorno inmediato (Chapa et alii, 1984). En los niveles tardíos se documentan (3) tanto Vitis vinifera como pólenes correspondientes a olivo (López, 1984), que se complementan con el hallazgo casual de una prensa de aceite en las campañas antiguas (Blanco, 1962). Los restos carpológicos señalan a la cebada vestida ( $\mathrm{Hor}$ deum vulgare) como especie de cereal mejor representada, mostrando indicios de un proceso previo de trilla y aventado. También hay evidencias de trigo, avena, legumbres (Vicia sativa) y ciertas leguminosas de difícil precisión. Los restos de fauna indican una diversidad de animales domésticos, destacando ovicápridos y cerdos, junto a un empleo frecuente de la caza como complemento comprensible en un entorno con un rico componente forestal.

La situación de Castellones de Céal en un punto clave de la ruta que transita por el pasillo de Pozo Alcón ha sido vinculada desde su descubrimiento con la antigua Fraxinum, una mansio citada en el Itinerario deAntonino y situada entre Tugia y Bactara en la vía que desde Cástulo llevaba a Málaga (Fernández Chicarro, 1955b). Se trate o no del lugar indicado en las fuentes, el poblado domina un vado de paso obligado que ha sido empleado tradicionalmente en el sistema local de comunicaciones. Por tanto, no resulta descabellado pensar en el asentamiento de Los Castellones no sólo como una ex-

(3) El estudio carpológico ha sido realizado por Ana Arnanz (Instituto de Historia, CSIC)

T. P., 57, n. $^{\circ} 1,2000$ 
plotación estrictamente local, sino como un emplazamiento en el que no sería rara la presencia de viajeros. Por todo ello, resulta razonable la explotación intensiva del medio inmediato, recurriendo a todo tipo de productos, naturales y cultivados. La pesca fluvial supone en este sentido un recurso idóneo, ya que se cuenta con dos ríos en los que vivieron diversas especies económicamente rentables y que, con los medios adecuados, no presentaban grandes dificultades para su obtención.

Los plomos de red encontrados en el espacio A de la vivienda 1 son numerosos y suponen un peso considerable, lo que parece excluir que se trate de un esparavel, haciendo más plausible su lectura como correspondientes a un trasmallo, en el que también se insertaría la piedra perforada que actuaría como lastre principal. La presencia de más de 60 unidades revela que la longitud de la red sería como mínimo de $6 \mathrm{~m}$., lo que supera el ancho del arroyo de Céal y se aproxima a la anchura del Guadiana Menor, siempre considerando la dificultad que implican estos cálculos, teniendo en cuenta además que no se conoce el número exacto de lastres y que éstos se pueden disponer a mayor o menor distancia según las necesidades de la red. De los flotadores, que necesariamente deberían insertarse en el cordel superior, y que están habitualmente hechos en corteza de madera, no ha quedado resto alguno debido a la combustión del local. Lo mismo puede decirse de la propia red, que estaría hecha con lino o cáñamo.

Desconocemos si existía una tradición local en el empleo de este tipo de artes de pesca, y lo cierto es que no se han recuperado todavía instrumentos que puedan ponerse en relación con la manufactura de las redes, si bien sus características harían difícil su conservación. No resulta descabellado pensar, sin embargo, que al igual que se importan recipientes y contenidos de áreas alejadas, también se pudieran incorporar al yacimiento estas redes como objetos manufacturados, siendo más fácil repararlas localmente, así como añadir o reponer los plomos de lastre o los flotadores. En todo caso, la valoración y el estudio de los diversos sistemas de pesca fluvial en el mundo ibérico irán progresivamente detallando las diversas características de esta estrategia económica.

\section{AGRADECIMIENTOS}

Este trabajo no hubiera podido realizarse sin la eficaz colaboración de varias personas, a las que agradecemos sinceramente su ayuda. Debemos citar especialmente a Raimundo Jordán por las informaciones relativas a los sistemas tradicionales de pesca empleados en la zona del yacimiento, así como a $\mathrm{M}^{\mathrm{a}}$ Antonia Herradón, conservadora del Museo Nacional de Antropología por las facilidades otorgadas en la revisión de las colecciones de redes de pesca depositadas en ese Museo. X.L. Ladra nos ha hecho partícipes de sus interesantes trabajos sobre la pesca fluvial en Galicia, y finalmente, Luis deAmbrosio y Jose Ambrosio González, del Museo Nacional de Ciencias Naturales, han colaborado proporcionándonos información sobre la fauna piscícola actual del Guadiana Menor.

\section{BIBLIOGRAFÍA}

Agudo Torrico, J. (1992): “Tejido de los paños de mallas y enjabelgado de las redes corianas". Etnografía Española, 8: 61-88.

Alves, F.J.S.; Alveirinho Dias, J.M.; Rocha de Almeida, M.J.; Ferreira, O. y TABORdA, R. (1988-1989): “A armadilla de pesca da época romana descoberta na praia de Silvade (Espino)". O Arqueólogo Português serie IV, 6/7: 187-226.

Aranegui, C. (1996): "Los platos de peces y el más allá". En M.A. Querol y T. Chapa (eds): Homenaje al Profesor Manuel Fernández Miranda, I. Complutum Extra, 6. Universidad Complutense. Madrid: 401-414.

Ballester Tormo, I.; Fletcher Valls, D.; Pla Ballester, E.; Jordá Cerdá, F. y AlCÁcer Grau, J. (1954): Corpus Vasorum Hispanorum. Cerámica del Cerro de San Miguel de Liria. CSIC. Diputación Provincial deValencia. Madrid.

Bas Morales, R. (1955): La pesca en España. Instituto de Investigaciones Pesqueras. Barcelona.

Beltrán Lloris, M.; Ortiz Palomar, M.E. y Paz Peralta, J.A. (1999): "La vajilla relacionada con el vino". En $E l$ Vino en la Antigüedad Romana. SimposioArqueología del Vino. Serie Varia 4. Universidad Autónoma. Madrid: 129-200.

Blanco, A. (1962): "El aceite en los albores de la Historia de España". Oretania, 10: 138-148.

Bonet, H. (1995): El Tossal de Sant Miquel de Llíria. La antigua Edeta y su territorio. SIP. Diputación de Valencia.

Brinkhuizen, D.C.y Clason, A. (1986): Fish andArchaeology. Studies in osteometry, taphonomy, seasonality and fishing methods. B.A.R. (IS) 294. Oxford.

CASTEEL, W. (1976): Fish remains in Archaeology and paleo-environmental studies. Academic Press. LondresN. York.

CAZORLA (1751): ... según las Respuestas Generales del Catastro de Ensenada. Colección Alcabala del Viento 
53. Centro de Gestión Catastral y Cooperación Tributaria. Tabapress. Madrid.

Celestino Pérez, S. (1996): El Palacio Santuario de Cancho Roano V-VI-VII. Los sectores oeste, sur y este. Publicaciones Del Museo Arqueológico Provincial de Badajoz, 3. Madrid.

Clark, J.G.D. (1948): "Development of fishing in Prehistoric Europe".TheAntiquaries Journla, 28(1-2): 45-48.

Chapa, T.; Fernández, M.; Pereira, J.y Ruiz, A. (1984): "Análisis económico y territorial de Los Castellones de Ceal (Jaén)". Arqueología Espacial. Coloquio sobre Distribución y Relaciones entre los Asentamientos. 4: 223-240.

Chapa, T.; Pereira, J.; Madrigal, A. y Mayoral, V. (1998): La necrópolis ibérica de Los Castellones de Céal (Hinojares, Jaén). Consejería de Cultura. Junta de Andalucía. Universidad de Jaén. Sevilla.

Doadrio, I.; Elvira, B. y Bernat, Y. (eds.), (1991): Peces continentales españoles. Inventario y clasificación de zonas fluviales. ICONA. CSIC. Madrid.

DuHAMEl DE Monceau, M. (1769-1779): Traité général des pêches. Paris.

FERNÁNDEZ ChicARRo, C. (1955a): "ProspecciónArqueológica en los Términos de Hinojares y La Guardia I". Boletín del Instituto de Estudios Gienenses, II(6): 8999.

FERnÁndez ChiCARro, C. (1955B): "Descubrimiento de una necrópolis ibérica y posible localización de la antigua Fraxinum". Zephyrus, VI: 293-294.

Fernández Rodríguez, M.; LóPez Fernández, F.J.; MAdrIgal Belinchón, A. y Mayoral Herrera, V. (1994): "Aproximación al estudio etnoarqueológico del Guadiana Menor (Jaén)". Trabajos de Prehistoria, 51(1): 111-126.

FeugÈre, M. (1992): "Les instruments de chasse, de pêche et d'agriculture". Lattara, 5: 139-162.

FLETCHER, D. (1968): "Esquema general sobre economía del pueblo ibero". Saguntum. Papeles del Laboratorio de Arqueología de Valencia, 5: 43-53.

Frutos Reyes, G. de y MuÑoz Vicente, A. (1996): "La industria pesquera y conservera púnico-gaditana: balance de la investigación. Nuevas perspectivas". SPAL, 5: 133-165.

Gómez Caruana, F. y Díaz Luna, J.L. (1991): Guía de los peces continentales de la Península Ibérica. Libros Penthlaton. Madrid.

Gracia Alonso, F. (1981-1982): “Ordenación tipológica del instrumental de pesca en bronce ibero-romano". Pyrenae, 17-18: 315-328.

Gruvel, A. (1928): La pêche dans la Préhistoire, dans l'Antiquité et chez les peuples primitifs. Société d’Editions Géographiques, Maritimes Et Coloniales. Paris.

Gunda, B. (1984): The fishing culture in the world. Studies in Ethnology, Cultural Ecology and Folklore. 2 Vols. Academici Klado. Budapest.
Jimeno Martínez, A.; Berzosa del Campo, R.; De la Torre Echávarri, J.I.; Granda Rubio, R. y Flores FernáNDEZ, R. (1999): “Actividades económicas en Numancia”. XXIV Congreso Nacional de Arqueología, 4. Romanización y desarrollo urbano en la Hispania republicana. Instituto de Patrimonio Histórico. Cartagena: 799-808.

Ladra Fernández, X.L. (1998): “Os Caneiros de Portomarín: achega a un peculiar e desaparecido sistema de pesca da anguía no río Minho". Cuadernos de Estudios Gallegos XLV (110): 257-286.

Ladra Fernández, X.L. y Pereiras Magariños, B. (e.p.). "Achega histórico-etnográfica ás pesqueiras do Ulla". VIII Semana Galega da Historia. Galicia Mare Nostrum: a importancia do mar en Galicia. Museo do Pobo Galego. Santiago de Compostela.

LE Galle, O. (1984): L'ichtiofaune d'eau douce dans les sites préhistoriques. Cahiers Du Quaternaire, 8. CNRS. Paris.

LÓPEZ, P. (1984): “Análisis polínico”. En T. Chapa, M. Fernández, J. Pereira y A. Ruiz (1984): Análisis económico y territorial de Los Castellones de Ceal (Jaén). Arqueología Espacial. Coloquio sobre Distribución y Relaciones entre los Asentamientos, 4: 223-240.

LoREnZo FERnÁndeZ, X. (1979): "Etnografía. Cultura Material". En R. Otero Pedrayo (dir.): Historia De Galiza. Akal. Madrid: 414-433.

Madoz, P. (1988): Diccionario Geográfico-EstadísticoHistórico de España y sus Posesiones de Ultramar (1845-50). 16 vols. Edición Facsímil. Ed. Ámbito. Valladolid.

MAYoral, V. (1996): “El hábitat ibérico tardío de Los Castellones de Ceal: organización del espacio y estructura socio-económica". Complutum, 7: 225-246.

MELCón LóPEZ, L. (1964): Métodos y artes de pesca en las aguas continentales españolas. Servicio Nacional de Pesca Fluvial y Caza. Serie Piscícola, 4. Madrid.

Merino, J.M. (1986): La pesca desde la Prehistoria hasta nuestros días (La pesca en el Pais Vasco). Servicio Central de Publicaciones del Gobierno Vasco. Vitoria.

Mız, H. (1986): "Die Tierknochenfunde aus drei argarzeitlichen Siedlungen in der Provinz Granada (Spanien)". Studien Über Frühe Tierknochenfunde Von Der Iberischen Halbinsel, 10. Deusches Archäologisches Institut. Abteilung Madrid. Munich.

Mora Aliseda, J. (1988): La pesca fluvial en Extremadura. Un modo de vida. Cuadernos Populares, 22. Junta de Extremadura. Mérida.

Navarro Palazón, J. y Robles Fernández, A. (1996): Liétor. Formas de vida rurales en Sarq al-Andalus a través de una ocultación de los siglos X-XI. Centro de Estudios Árabes y Arqueológicos “IbnArabi”. Murcia.

Navas, J.M. (1945): Pesca marítima.Artes de pesca, embarcaciones, pesquerías, industrias. Publicaciones del Instituto Social de la Marina. Madrid.

Pardo, L. (1950): Apuntes para la Historia de la Pesca

T. P., 57, n. ${ }^{\circ} 1,2000$ 
Continental Española. Tomo I: desde los tiempos remotos al siglo XVIII. Ministerio de Agricultura. Madrid.

PicAzo, J. y Alba-Tercedor, J. (1996): “Caracterización físico-química de las aguas de la cuenca del río Guadiana Menor". IV Simposio sobre el Agua en Andalucía (Almería, 1996). Vol II. Instituto Tecnológico Geominero de España. Madrid: 155-164.

Powell, J. (1996): Fishing in the Prehistoric Aegean. Paul Astroms Forlag. Jonsered.

Puche, I. (1959): Pesca Fluvial. Temas Españoles 330. Publicaciones Españolas. Madrid.

PulaK, C. (1988): "A Bronze Age Shipwreck at Ulu Burun, Turkey: 1985 Campaign”. American Journal of Archaeology, 92(1): 1-38.

Py, M. (1991): "Catalogue des petits objets". En Exploration de la Ville Portuaire de Lattes. Serie Lattara.

RADCLIFFE, W. (1926): Fishing from the Earliest Times. John Murray. Londres.

RAU, C. (1884): Prehistoric fishing in Europe and North
America. Smithsonian Contributions to Knowledge, 509. Washington.

Romero Díaz, M. A. (1989): Las Cuencas de los ríos Castril y Guardal (Cabecera del Guadalquivir). Estudio hidrogeomorfológico. Excmo. Ayuntamiento de Huéscar (Granada). Universidad de Murcia.

Roselló, E. y Morales, A. (1994): Castillo de Doña Blanca. Archaeoenvironmental Investigations in the Bay of Cádiz, Spain (750-500 BC). B.A.R. (IS) 593. Oxford.

Sáñez Reguart, A. (1791): Diccionario Histórico de los Artes de la Pesca Nacional. Ministerio de Agricultura, Pesca y Alimentación (1988). Madrid.

STERnBerg, M. (1995): La pêche à Lattes dans l'Antiquité a travers de l'analyse de l'ichtyofaune. Lattara, 8.

Thomazi, A. (1947): Histoire de la pêche des âges de la pierre à nos jours. Payot. Paris.

Veiga Ferreira, O. da (1968): "Algumas notas acerca da pesca na Antiguidade". O Arqueólogo Português, Serie III, 2: 113-133. 